Гендерные особенности метаболических нарушений у пожилых больных с сердечной недостаточностью с промежуточной фракцией выброса

\author{
Е.В. Гостева (1) \\ Федеральное государственное бюджетное образовательное учреждение высшего \\ образования «Воронежский государственный медицинский университет им. Н.Н. Бурденко» \\ Министерства здравоохранения Российской Федерации, \\ ул. Студенческая, д. 10, г. Воронеж, 394036, Российская Федерация \\ Автор для переписки: Е.В. Гостева (pvb.gosteva@mail.ru)
}

Резюме

Актуальность: На сегодняшний день важным является увеличение частоты развития сердечной недостаточности с возрастом. Патогенетические механизмы, выявляемые при метаболическом синдроме и атеросклерозе свидетельствуют о взаимосвязи этих процессов, при этом у каждого второго больного с коронарной патологией выявляются признаки метаболических нарушений. Клиническая значимость оценки метаболического синдрома и его отдельных компонентов в отношении прогнозирования риска развития сердечной недостаточности с промежуточной фракцией выброса $(\mathrm{CH} Ф В)$ ишемической этиологии остается неясной. Цель исследования: Выяснить взаимосвязь метаболического синдрома и его компонентов с риском развития СНпФВ у пожилых больных и выявить гендерные патофизиологические различия между больными СНпФВ пожилого возраста. Материалы и методы: Обследовано 120 больных СНпФВ (40-49\%) II функционального класса по NYHA ишемического ге-

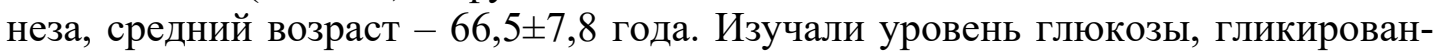
ного гемоглобина (HbA1c), инсулина, лептина в сыворотке крови, рассчитывали индекс HOMA-IR. Оценивали липидный спектр - уровень общего холестерина, липопротеидов низкой и высокой плотности, триглицеридов. Результаты: Выявлена заметная положительная корреляционная связь абдоминального ожирения с уровнем лептина $(\mathrm{r}=0,56, \mathrm{p}<0,05)$, с концентрацией в плазме крови инсулина $(\mathrm{r}=0,54, \mathrm{p}<0,05)$ и индексом HOMA-IR $(\mathrm{r}=0,53, \mathrm{p}<0,05)$. Так же выявлена высокая положительная связь абдоминального ожирения и уровня общего холестерина $(\mathrm{r}=0,86, \mathrm{p}<0,001)$, умеренная связь с уровнем триглицеридов $(\mathrm{r}=0,46, \mathrm{p}<0,05)$ и умеренная отрицательная корреляционная связь с липопротеидами высокой плотности $(\mathrm{r}=-0,34, \mathrm{p}<0,05)$. Изучение гендерных особенностей у больных СНпФВ на фоне метаболического синдрома позволило выявить бо- 
лее высокую концентрацию инсулина $(\Delta 17 \%)$, лептина $(\Delta 33 \%)$ в крови у женщин, чем мужчин. У женщин выявлено более высокое содержание липопротеидов низкой плотности $(\Delta 12 \%)$ и более низкое содержание липопротеидов высокой плотности $(\Delta 10 \%)$, чем у мужчин. Заключение: Таким образом, у женщин с СНпФВ ишемического генеза на фоне метаболического синдрома отмечается наиболее выраженное по сравнению с мужчинами абдоминальное ожирение, ассоциированное с более высокими уровнями лептина крови и инсулинорезистентности, более высоким уровнем липопротеидов низкой плотности и более низким содержанием липопротеидов высокой плотности.

Ключевые слова: сердечная недостаточность; промежуточная фракция выброса; пожилой возраст; метаболический синдром

Для цитирования: Гостева ЕВ. Гендерные особенности метаболических нарушений у пожилых больных с сердечной недостаточностью с промежуточной фракцией выброса. Научные результаты биомедицинских исследований. 2020;6(2):249-260. DOI: 10.18413/2658-6533-2020-6-2-0-9

\title{
Gender features of metabolic disorders in elderly patients with heart failure with mid-range ejection fraction
}

\author{
Elena V. Gosteva \\ Burdenko Voronezh State Medical University, \\ 10 Studencheskaya St., Voronezh, 394036, Russia \\ Corresponding author: Elena V. Gosteva (pvb.gosteva@mail.ru)
}

\begin{abstract}
Background: Today, it is important to pay special attention to the increased incidence of heart failure developing with age. Pathogenetic mechanisms detected in metabolic syndrome and atherosclerosis indicate the relationship of these processes, while every second patient with cardiovascular disease shows signs of metabolic syndrome. The clinical significance of the assessment of metabolic syndrome and its individual components to predict the risk of development of heart failure with midrange ejection fraction (HFmrEF) ischemic etiology remains unclear. The aim of the study: To find out the relationship between metabolic syndrome and its components with the risk of developing HFmrEF in elderly patients and to identify gender pathophysiological differences between elderly HFmrEF patients with metabolic syndrome. Materials and methods: 120 patients with HFmrEF (40-49\%) of functional class II according to NYHA were examined, the age was $66.5 \pm 7.8$ years. We studied the level of glucose, glycated hemoglobin, insulin, and leptin in the blood serum, and calculated the HOMA-IR index. The lipid spectrum was assessed - the level of total cholesterol, low-and high-density lipoproteins (LDL, HDL-C), and triglycerides. Results: There was a noticeable positive correlation between abdominal obesity and leptin levels $(r=0.56, p<0.05)$, with the concentration of insulin $(r=0.54, p<0.05)$ and the HOMA-IR index $(\mathrm{r}=0.53, \mathrm{p}<0.05)$. There was also a high positive association between abdominal obesity and the level of total cholesterol $(r=0.86, p<0.001)$, a mod-
\end{abstract}


erate association with the level of triglycerides $(\mathrm{r}=0.46)$, and a moderate negative correlation with HDL $(r=-0.34, p<0.05)$. The study of gender characteristics of metabolic syndrome in patients with HFmrEF revealed a higher concentration of insulin $(\Delta 17 \%)$, leptin $(\Delta 33 \%)$ in the blood of women than in men. Women demonstrated a higher content LDL $(\Delta 10 \%)$ and lower content of HDL-C $(\Delta 12 \%)$, compared to men. Conclusion: Women with HFmrEF on the background of metabolic syndrome have the most pronounced abdominal obesity compared to men, associated with higher levels of blood leptin and insulin resistance, higher levels of LDL cholesterol and lower levels of HDL.

Keywords: heart failure; mid-range ejection fraction; old age; metabolic syndrome

For citation: Gosteva EV. Gender features of metabolic disorders in elderly patients with heart failure with mid-range ejection fraction. Research Results in Biomedicine. 2020;6(2):249-260. Russian. DOI: 10.18413/2658-6533-2020-6-2-0-9

Введение. Сердечно-сосудистые заболевания являются наиболее важной причиной заболеваемости и смертности среди пожилых людей во всем мире, при этом сердечная недостаточность является наиболее быстро растущей сердечнососудистой нагрузкой на здоровье во всем мире $[1,2]$. Частота сердечно-сосудистых событий увеличивается после 65 лет у мужчин и после 75 лет у женщин [2], при этом у каждого второго больного с коронарной патологией выявляются признаки метаболического синдрома (МС) [3]. Пожилые пациенты представляют собой особую категорию пациентов, обусловленную физиологическими изменениями, вызванными возрастом, большим количеством сопутствующих заболеваний и медикаментозным лечением. По данным Всемирной Организации Здравоохранения, пожилые люди находятся в возрасте более 60-65 лет. Принимая во внимание большой процент пожилых людей в общей численности населения, очень важно отметить, что частота развития сердечной недостаточности увеличивается с возрастом. Клиническая значимость оценки МС и его отдельных компонентов в отношении прогнозирования риска развития сердечной недостаточности с промежуточной фракцией выброса ишемической этиологии остается неясной.

Цель исследования. Выявить гендерные патофизиологические различия между больными пожилого возраста с СНпФВ в зависимости от наличия МС.
Материалы и методы исследования. В исследовании приняли участие 120 больных ХCH с промежуточной фракцией выброса (40-49\%) II функционального класса по NYHA ишемического генеза. Симптомы и признаки ХCH определялись согласно рекомендациям экспертного комитета Европейского общества кардиологов и национальным рекомендациям, 2018 года. Критерием исключения были онкологические, инфекционные заболевания, тяжелые поражения печени и почек в анамнезе; любые хирургические вмешательства в предшествующие 6 месяцев. Все больные подписывали информированное согласие.

У всех пациентов была проведена оценка состояния их здоровья путем физикального осмотра и изучения медицинской документации. Мы оценили возраст больных, наличие метаболических нарушений, а также корреляцию между изучаемыми показателями.

Определение показателей углеводного обмена проводили всем больным, уровень глюкозы изучали автоматическим анализатором (Vital Scientific, Нидерланды); гликированного гемоглобина (HbA1c) в крови непосредственным определением; инсулин - автоматически на анализаторе Immulite 2000 (США). По формуле D. Matthews (1985) рассчитывали индекс HOMA-IR (показатель инсулинорезистентности). Уровень лептина определяли иммуноферментным методом с использо- 
ванием систем Leptin ELISA (Diaghostics Biochem Canada Inc (DHC Inc)).

Изучение липидного спектра заключалось в определении уровня общего холестерина (OX) на автоматическом биохимическом анализаторе (Vital Scientific, Нидерланды). Холестерин липопротеинов высокой плотности (ЛПВП) и триглицериды (ТГ) количественно оценивали прямым гомогенным тестом (Human GmbH, Германия). Уровень липопротеинов низкой методом по формуле W. Friedewald (1972 г.)

Статистический анализ проводился с использованием STATISTICA 10.0, формирование базы данных пациентов вели с помощью таблиц Excel MS Office 2016. Исходные и полученные значения были описаны с использованием стандартной статистики и сравнены с помощью парных $t$-тестов или по Вилкоксону. Параметры значений представлены в виде среднего показателя со стандартным отклонением или медианы (Me) и интерквартильные размахи $\left(\mathrm{Q}_{25}\right.$, $\left.\mathrm{Q}_{75}\right)$. Корреляции между двумя переменными оценивались с помощью рангового критерия Спирмена. Стаплотности (ЛПНП) определяли расчетным

тистически достоверными считали различия при $\mathrm{p}<0,05$.

Результаты и их обсуждение. Общую группу (120 пациентов) составили $65(54 \%)$ мужчин и $55(46 \%)$ женщин в возрасте от 58 до 74 лет (средний возраст $66,5 \pm 7,8$ года).

В нашем исследовании все пациенты были разделены на две группы в зависимости от наличия метаболического синдрома. Симптомы и признаки ХСН определялись согласно рекомендациям экспертного комитета Европейского общества кардиологов и национальным рекомендациям, 2018 года.

Больные хронической сердечной недостаточностью с промежуточной фракцией выброса на фоне ИБС представляют интерес в изучении особенностей клиникоанамнестической, антропометрической, функциональной характеристики и метаболических особенностей, так как литературные данные по клиническому сочетанию этих заболеваний отсутствуют.

Сравнительный анализ клинической характеристики обследованных групп представлен в таблице 1 .

Таблийа 1

\section{Клиническая характеристика обследованных групп}

Table 1

Clinical characteristics of the examined groups

\begin{tabular}{|c|c|c|}
\hline Клинические данные & $\begin{array}{c}\text { Группа 1 } \\
\text { СНпФВ, ИБС, без МС }\end{array}$ & $\begin{array}{c}\text { Группа } 2 \\
\text { СНпФВ, ИБС, на фоне МС }\end{array}$ \\
\hline Всего обследовано, чел. (\%) & $50(100)$ & $70(100)$ \\
\hline $\begin{array}{l}\text { мужчин, чел. (\%) } \\
\text { женщин, чел. (\%) }\end{array}$ & $\begin{array}{l}30(60)^{*} \\
20(40)\end{array}$ & $\begin{array}{l}32(46) \\
38(54)^{*}\end{array}$ \\
\hline Средний возраст, лет & $65,8 \pm 7,1$ & $66,9 \pm 6,7$ \\
\hline $\begin{array}{l}\text { ИБС: стенокардия, ФК: } \\
\text { Чел. (\%) }\end{array}$ & $50(100)$ & $70(100)$ \\
\hline 0, чел. (\%) & $7(14)$ & $5(7)$ \\
\hline I, чел. (\%) & $29(58) * *$ & $14(20)$ \\
\hline II, чел. (\%) & $14(28)$ & $51(73) * *$ \\
\hline НК по NYHA, чел. (\%) & $50(100)$ & $70(100)$ \\
\hline I ФК, чел. (\%) & $27(54) * *$ & $21(26)$ \\
\hline II ФК, чел. (\%) & $23(46)$ & $59(74)^{* *}$ \\
\hline$\Phi B$ в $\%, M \pm m$ & $48,3 \pm 1,6$ & $44,1 \pm 2,8$ \\
\hline
\end{tabular}

Примечание: * $\mathrm{p}<0,05 ; * * \mathrm{p}<0,01-$ по исследуемому показателю между группами пациентов Note: $* \mathrm{p}<0.05 ; * * \mathrm{p}<0.01-$ for the studied indicator between groups of patients 
Кроме того, больные с СНпФВ на фоне МС чаще имели артериальную гипертензию (88\%) по сравнению с больными без МС (48\%), пароксизмальную фибрилляцию предсердий $8 \%$ и $5 \%$ соответственно.

Нами проанализированы клиниколабораторные данные больных обеих групп СНпФВ ишемического генеза (без $\mathrm{MC}$ и при наличии $\mathrm{MC)}$ в зависимости от пола: подгруппа 1.1 - мужчины СНпФВ ишемического генеза без метаболических нарушений; подгруппа 1.2 - женщины СНпФВ ишемического генеза без метаболических нарушений; подгруппа 2.1 - мужчины СНпФВ ишемического генеза с метаболическим синдромом; подгруппа 2.2 - женщины СНпФВ ишемического генеза с метаболическим синдромом. В клинической картине СНпФВ женщины по сравнению с мужчинами чаще имели пастозность голеней и стоп, одышку при физической нагрузке, патологический III тон, набухание яремных вен.

Основным диагностическим критерием метаболического синдрома, в соответствии с рекомендациями (2009), является абдоминальное ожирение. В связи с этим при диагностике абдоминального ожирения мы использовали окружность талии (ОТ), как основной критерий, и отношение окружности талии к окружности бедер (ОБ). Объем талии у больных СНпФВ без МС составил 97,2 $(95,1 ; 100,3)$ см, у больных СНпФВ ишемического генеза и МС был выше - $116,3(107,3 ; 127,1)$ см, при этом разница $(\Delta \%)$ составила $16 \%$ $(\mathrm{p}<0,01)$. Показатель ОТ/ОБ у больных СНпФВ без $\mathrm{MC} \mathrm{-} \mathrm{0,96}(0,93 ; 0,99)$, у больных СНпФВ, ИБС на фоне МС - 1,09 (1,04; $1,17)$, разница составила $10 \%(\mathrm{p}<0,05)$.

Объем талии у мужчин в подгруппе 1.1 - 94,9 $(90,5 ; 98,3)$ см, у женщин в подгруппе 1.2 составил 95,8 $(85,2 ; 97,4)$ см. Достоверной разницы по объему талии между мужчинами и женщинами СНпФВ, ИБС без МС не выявлено. Объем талии у мужчин в подгруппе $2.1-108,3(107,3 ; 111,8)$ см, у женщин в подгруппе $2.2-121,4$ $(114,3 ; 127,1)$ см и был достоверно выше, чем у мужчин $(\Delta 11 \%, \mathrm{p}<0,05)$. Отношение ОТ/ОБ у мужчин в подгруппе $1.1-0,99$ $(0,94 ; 1,05)$, у женщин в подгруппе 1.2 $0,95(0,87 ; 1,02)$, что свидетельствует об отсутствии достоверных различий у больных без МC, $\Delta \%$ составила $4 \%(\mathrm{p}>0,05)$. Отношение ОТ/ОБ у мужчин в подгруппе 2.1 - 1,05 (1,04; 1,07), у женщин в подгруппе $2.2-1,14(1,09 ; 1,17)$, при этом $\Delta \%$ составила $8 \%(\mathrm{p}<0,05)$, что согласуется с опубликованным эпидемиологическим исследованием [4], в котором у женщин с метаболическим синдромом определялись более высокие показатели окружности талии.

По данным литературы, при МС абдоминальное ожирение связано с повышенной продукцией лептина и развитием лептинорезистентности [5]. Было показано, что лептин обладает защитными эффектами, связанными с ослаблением окислительного стресса. В связи с этим, нами было изучено содержание в крови данного показателя у больных СНпФВ. Содержание лептина в крови у больных СНпФВ без нарушений метаболизма - 13,6 $(10,5 ; 18,4)$ нг/мл, у больных СНпФВ ишемической этиологии с МС - 26,6 (17,8; 32,7) нг/мл, разница составила $49 \%(\mathrm{p}<0,001)$. Кроме того, полученные нами результаты свидетельствуют о достоверно более высоком уровне лептина в крови у женщин в обеих подгруппах, чем у мужчин (рис. 1). Уровень лептина у мужчин в подгруппе 1.1 $11,9(9,2 ; 12,9)$ нг/мл, у женщин в подгруппе 1.2 - 14,7 $(12,4 ; 17,1)$ нг/мл, при этом $\Delta \%$ составила $19 \%(\mathrm{p}<0,01)$. Уровень лептина у мужчин в подгруппе $2.1-19,2$ $(16,8 ; 22,4)$ нг/мл, у женщин в подгруппе $2.2-28,7(24,6 ; 34,1)$ нг/мл, при этом $\Delta \%$ составила $33 \%(\mathrm{p}<0,01)$.

Таким образом, наличие МС у пожилых больных с СНпФВ ишемического генеза ассоциировалось с абдоминальным ожирением, более выраженном у женщин, чем у мужчин. В патогенезе метаболических нарушений у больных СНпФВ ишемического генеза имеет место гиперлептинемия, более выраженная у женщин, чем у мужчин. 
У больных с метаболическими нарушениями особое значение имеет состояние инсулинорезистентности, для диагностики которого нами был использован расчетный индекс HOMA-IR, определенный по показателям глюкозы натощак и содержания инсулина в крови.

В группе больных СНпФВ без МС уровень инсулина в крови - 8,86 $(8,64$; $9,25)$ мкМЕ/мл, у больных группы СНпФВ на фоне $\mathrm{MC}-14,02 \quad(11,01 ; 17,24)$ мкME/мл, то есть выше на $(\Delta \%)-36 \%$ $(\mathrm{p}<0,01)$. У мужчин в подгруппе 1.1 уро- вень инсулина в крови - 9,07 $(9,22 ; 9,75)$ мкMЕ/мл, у женщин в подгруппе 1.2 (рис.2) выявлены более высокие значения данного показателя - 10,02 $(9,91 ; 10,09)$ мкМЕ/мл по сравнению с мужчинами, при этом $\Delta \%$ составила $9 \%(\mathrm{p}<0,05)$. У мужчин в подгруппе 2.1 уровень инсулина в крови - 13,25 $(11,01 ; 15,38)$ мкМЕ/мЛ, у женщин в подгруппе 2.2 выявлены более высокие уровни инсулина в крови $(16,01$ $(15,81 ; 17,24)$ мкМЕ/мЛ) по сравнению с мужчинами, $\Delta \%$ составила $17 \%(\mathrm{p}<0,01)$.

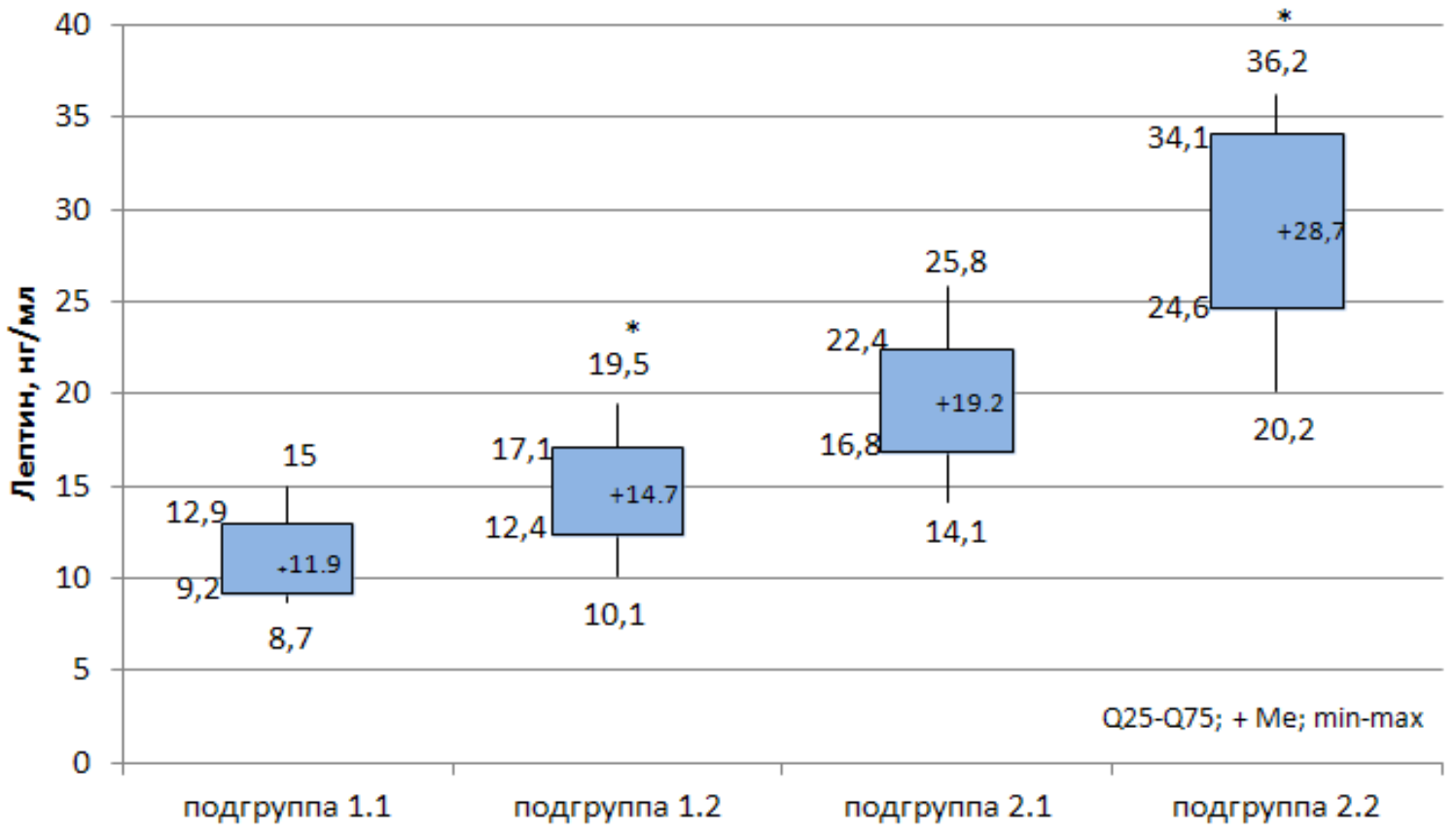

Рис. 1. Уровни лептина в изучаемых подгруппах пожилых больных $\mathrm{CHпФВ,}{ }^{*} \mathrm{p}<0,05$ между подгруппами женщин и мужчин

Fig. 1. Leptin levels in the studied subgroups of elderly patients HFmrEF, ${ }^{*} p<0.05$ between subgroups of women and men

В группе пожилых больных СНпФВ без МС уровень глюкозы находился в пределах нормальных значений и составил $5,32(5,10 ; 5,44)$ ммоль/л, в группе больных СНпФВ на фоне $\mathrm{MC}-6,08(5,78 ; 6,22)$ ммоль/л, разница составила $13 \%(\mathrm{p}<0,05)$. Гендерных различий по уровню глюкозы выявлено не было.

Среди критериев метаболического синдрома активно обсуждается роль инсулинорезистентности, которая является компонентом метаболического синдрома и была определена нами на основании ин- декса HOMA-IR, рассчитанного из показателей глюкозы натощак и концентрации инсулина в крови. Инсулинорезистентность сопровождается компенсаторным повышением секреции инсулина и гиперинсулинемией для поддержания нормальной концентрации глюкозы.

У больных СНпФВ ишемического генеза без метаболических нарушений значение индекса HOMA-IR было ниже диагностического критерия инсулинорезистентности и составило - 2,52 $(2,14 ; 2,72)$ ед., у больных же в группе СНпФВ с нали- 
чием МС индекс HOMA-IR был 4,92 (4,61; $5,28)$ ед., разница $(\Delta \%)$ составила $48 \%$ $(\mathrm{p}<0,001)$, что является косвенным признаком инсулинорезистентности. При изучении гендерных особенностей индекса HOMA-IR было установлено, что у мужчин в подгруппе 1.1 значение составило
$2,31(2,05 ; 2,47)$ ед., у женщин в подгруппе 1.2 - 2,65 $(2,47 ; 2,77)$ ед., что выше на $13 \%$ $(\mathrm{p}<0,05) . \quad$ В группе пожилых мужчин СНпФВ на фоне МС, его значение было $4,32(4,11 ; 4,58)$ ед., у женщин (подгруппа $2.2)-5,58(5,32 ; 5,74)$ ед., разница составила $23 \%(\mathrm{p}<0,01)$.

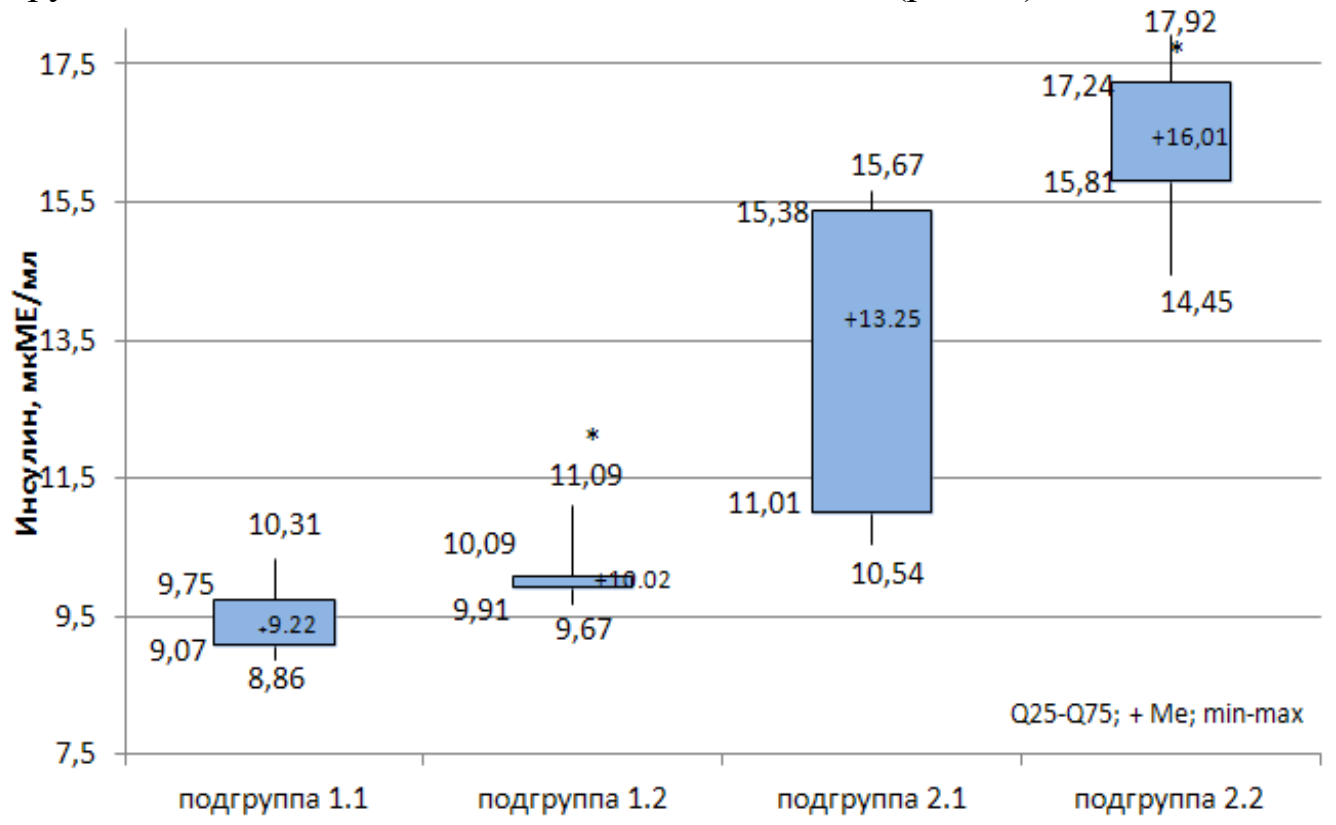

Рис. 2. Уровень инсулина в изучаемых подгруппах пожилых больных СНпФВ; *p<0,05 между подгруппами женщин и мужчин

Fig. 2. The level of insulin in the studied subgroups of elderly patients HFmrEF; ${ }^{*} \mathrm{p}<0.05$ between subgroups of women and men

Соответственно, у больных СНпФВ с МС повышен как уровень глюкозы, так и инсулина, что свидетельствует об инсулинорезистентности. Различия по индексу HOMA-IR между группами больных СНпФВ ишемического генеза в зависимости от МС определялись в наибольшей степени уровнем инсулина, чем глюкозы.

Результаты нашей работы свидетельствуют, что независимо от наличия метаболического синдрома, пожилые женщины с СНпФВ ишемического генеза имеют более высокие уровни инсулина и индекса HOMA-IR, чем мужчины.

C учетом того, что причиной развития сердечной недостаточности у пожилых больных в нашем исследовании была ишемическая болезнь сердца, мы оценили гендерные особенности и зависимость содержания ОХ от наличия МС. У больных
СНпФВ, имеющих метаболические нарушения уровень общего холестерина (OX) крови составил $6,68(6,38 ; 6,96)$ ммоль/л, что превышает показатели группы сравнения на $17 \%(\mathrm{p}<0,01)$. У больных СНпФВ без МС содержание ОX - 5,52 $(5,10 ; 5,79)$ ммоль/л. У мужчин в подгруппе 1.1 уровень холестерина в плазме крови составил $5,58(5,11 ; 5,72)$ ммоль/л, у женщин в подгруппе 1.2 - 5,51 (4,96; 5,68) ммоль/л $(\mathrm{p}>0,05)$. У женщин в подгруппе 2.2. концентрация $\mathrm{OX}$ была $6,72(6,53 ; 6,94)$, у мужчин в подгруппе $2.1-6,67(6,38 ; 6,96)$ ммоль/л ( $>0,05)$. Достоверных гендерных различий по содержанию общего холестерина нами не установлено. Нами проанализировано содержание ТГ в обеих группах больных СНпФВ и выявлено, что в группе больных СНпФВ с МС уровень ТГ был выше, чем в группе без МС (соответственно, 2,38 $(2,21 ; 2,54)$ ммоль/л и 1,76 
$(1,54 ; 1,88)$ ммоль/л, разница составила $26 \%(\mathrm{p}<0,01)$. Достоверных различий по гендерному признаку выявлено не было.

В группе пожилых больных СНпФВ на фоне МС уровень ЛПНП был 3,62 (3,34; $3,86)$ ммоль/л, а в группе сравнения без
МC - 2,85 $(2,76 ; 3,30)$ ммоль/л, разница составила $21 \%(\mathrm{p}<0,01)$. Особый интерес у пожилых больных представляют гендерные особенности содержания в крови высоко атерогенного ЛПНП, что представлено на рисунке 3.

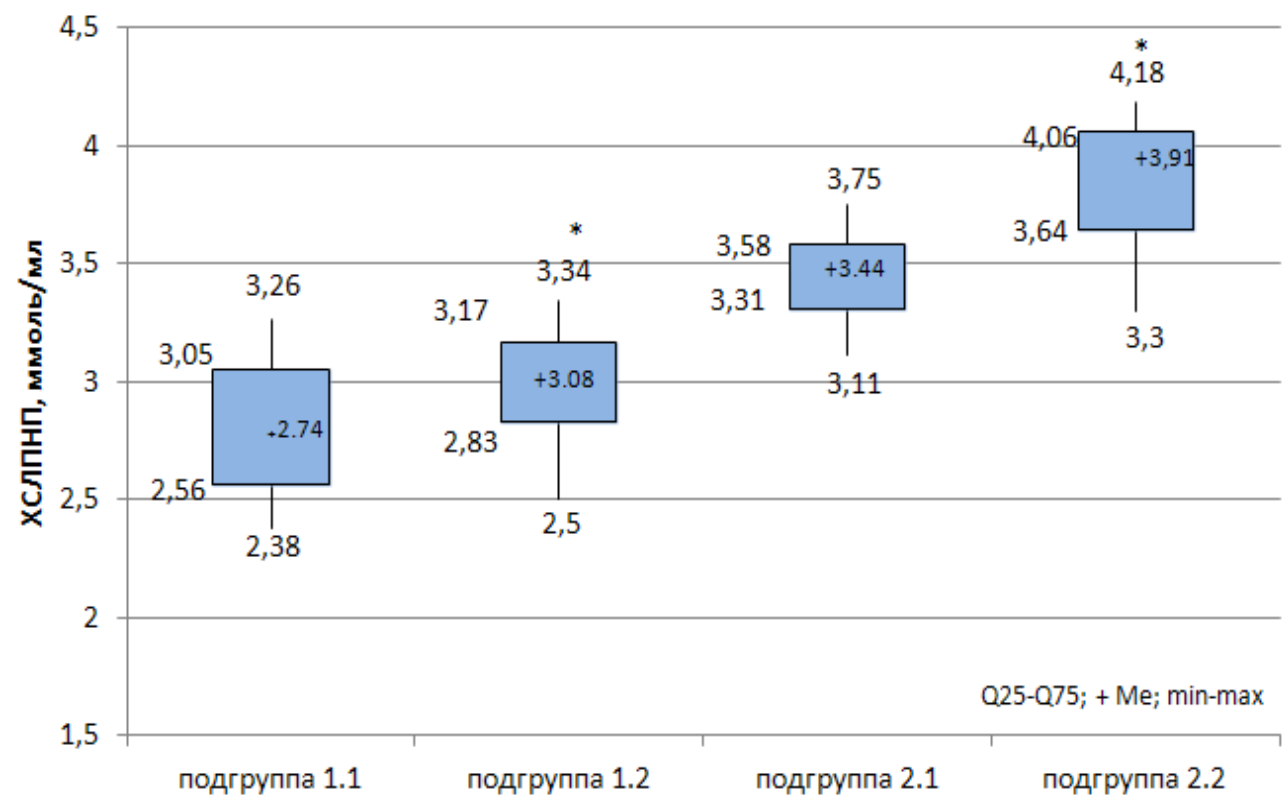

Рис. 3. Содержание ЛПНП в изучаемых группах больных СНпФВ *p $<0,05$ между подгруппами женщин и мужчин

Fig. 3. Content of LDL cholesterol in the studied groups of patients with HFmrEF $* p<0.05$ between subgroups of women and men

Так, у пожилых мужчин (подгруппа 1.1) с СНпФВ без МС концентрация ХС ЛПНП в крови была - 2,74 $(2,56 ; 3,05)$ ммоль/л, в то время как у женщин подгруппы 1.2 она составила $3,08(2,83 ; 3,17)$ ммоль/л, что на $\Delta 11 \%$ выше, чем у мужчин $(\mathrm{p}<0,05)$. Следует отметить, что у пожилых женщин на фоне МС (подгруппа 2.2) содержание ЛПНП в плазме крови составило - 3,91 $(3,64 ; 4,06)$ ммоль/л, при этом у мужчин концентрация - 3,44 $(3,31 ; 3,58)$ ммоль/л, что на $12 \%$ ниже, чем у женщин $(\mathrm{p}<0,05)$.

Известно, что при МС имеет место дисбаланс между про- и антиатерогенными фракциями липидов. В связи с этим мы проанализировали концентрацию ЛПВП в плазме крови изучаемых больных. В группе пожилых больных СНпФВ без МС содержание ЛПВП составило 1,12 (1,02; $1,15)$ ммоль/л, в группе больных на фоне
МС выявлен более низкий уровень липопротеидов высокой плотности - всего 0,90 $(0,78 ; 0,96)$ ммоль/л, разница между группами составила $\Delta 20 \%(\mathrm{p}<0,01)$.

Не меньший интерес представляют гендерные различия антиатерогенной фракции липопротеидов у пожилых больных. Так, у мужчин подгруппы 1.1 содержание в крови ЛПВП - $1,07(1,00 ; 1,16)$ ммоль/л, в то время как у женщин подгруппе $1.2-1,17(1,10 ; 1,22)$ ммоль/л, разница составила $8 \%(\mathrm{p}<0,05)$. Следует обратить внимание, что при сочетании СНпФВ и МС у пожилых женщин уровень липопротеидов высокой плотности оказался ниже $(0,81(0,78 ; 0,85)$ ммоль/л), чем у мужчин - $0,90(0,85 ; 0,96)$ ммоль/л, разница $(\Delta, \%)$ между группами составила $10 \%$ $(\mathrm{p}<0,05)$.

Таким образом, проанализировав гендерные изменения липидного профиля 
у пожилых больных СНпФВ ишемического генеза в зависимости от наличия МС можно сделать вывод, что они состоят в более высоком уровне ЛПНП и более низком уровне ЛПВП у женщин, что ассоциируется с дополнительным усилением атерогенного потенциала крови по сравнению с мужчинами.

Нами был проведен анализ взаимосвязи абдоминального ожирения (по критерию ОТ) с изученными показателями метаболического статуса у пожилых больных СНпФВ ишемического генеза. Нами выявлена заметная положительная корреляционная связь абдоминального ожирения с уровнем лептина $(\mathrm{r}=0,56, \mathrm{p}<0,05)$, что полностью согласуется с литературными данными об избыточной продукции лептина при МС [6]. Лептинорезистентность, в свою очередь, приводит к ожирению [7].

Кроме того, нами выявлена заметная положительная корреляционная зависимость абдоминального ожирения с концентрацией в плазме крови инсулина $(\mathrm{r}=0,54$, $\mathrm{p}<0,05)$ и индексом HOMA-IR ( $\mathrm{r}=0,53$, $\mathrm{p}<0,05)$, то есть развитием инсулинорезистентности, которая на сегодняшний день так же относится к основным компонентам МС. Известно, что гиперинсулинемия приводит к снижению содержания липопротеидов высокой плотности, изменению структуры и размеров липопротеидов, ремоделированию макро- и микрососудистого русла $[8,9]$. Кроме того, известно, что при МС повышенное содержание инсулина в крови при нарушенной толерантности к глюкозе с одной стороны поддерживает нормогликемию, с другой - ведет к нарушениям, способствующим развитию сердечно-сосудистых заболеваний $[10,11]$.

В результате проведенного корреляционного анализа нами выявлена высокая положительная связь абдоминального ожирения и уровня $\mathrm{OX}(\mathrm{r}=0,86, \mathrm{p}<0,001)$, умеренная связь с уровнем ТГ $(\mathrm{r}=0,46$ $\mathrm{p}<0,05)$ и умеренная отрицательная корреляционная связь с ЛПВП $(\mathrm{r}=-0,34, \mathrm{p}<0,05)$. Известно, что гиперхолестеринемия является ведущей причиной ИБС [12]. В нашем исследовании продемонстрировано, что на фоне МС у пожилых больных СНпФВ ишемического генеза увеличивается атерогенность плазмы крови за счет более высокого содержания ОХ и ТГ по сравнению с пожилыми больными без МС. Дислипидемия у пожилых больных СНпФВ ишемического генеза усугубляется за счет более высокой концентрации ЛПНП и более низкой ЛПВП на фоне метаболического синдрома. В соответствии с данными литературы, дислипидемия у больных с МС заключается в повышении в крови уровня триглицеридов и снижении липопротеидов высокой плотности на фоне снижения чувствительности тканей к инсулину [13].

Задачей исследования было изучение гендерных особенностей у пожилых больных СНпФВ ишемического генеза на фоне МС. Важным представляется установленная нами у пожилых женщин с СНпФВ, ИБС связь абдоминального ожирения с ОТ, которая у них была на $11 \%$ больше, чем у мужчин.

В литературе [14] описан более высокий уровень лептина у женщин, чем у мужчин, что объяснялось особенностями распределения жира в женском организме, стимулирующим эффектом эстрогенов и прогестерона или подавляющим влиянием андрогенов. В другом опубликованном исследовании [15] было показано, что у женщин с МС гиперлептинемия тесно коррелирует с инсулинорезистентностью. Однако, в этих исследованиях не изучались гендерные различия в зависимости от возрастной категории. В связи с этим, и так же учитывая литературные данные об участии лептина в развитии ожирения, мы провели оценку его концентрации в крови у пожилых больных по гендерному признаку. Так, нами выявлено, что у пожилых женщин с СНпФВ ишемического генеза на фоне МС уровень лептина в крови был выше $(\Delta, 33 \%)$, чем у мужчин.

Особенности углеводного обмена у мужчин и женщин представляют интерес для анализа, поскольку инсулинорезистентность является одной из кардинальных характеристик метаболического синдрома [16]. В нашей работе установлено, 
что при наличии СНпФВ на фоне МС у пожилых женщин по сравнению с мужчинами определялась более высокая $(\Delta, 17 \%)$ концентрация инсулина в крови.

При сравнительном анализе липидного спектра крови у мужчин и женщин мы не выявили статистически значимых различий по уровню общего холестерина и триглицеридов. Вместе с тем, были обнаружены особенности, связанные с уровнем в крови высоко атерогенных липопротеинов низкой плотности, уровень которых был у пожилых женщин выше $(\Delta, 12 \%)$, чем мужчин. В тоже время, показатель липопротеидов высокой плотности у пожилых женщин СНпФВ на фоне МС был ниже $(\Delta, 10 \%)$, чем у мужчин. При интерпретации полученных результатов мы учитывали, что женщины, включенные в данное исследование, находились в периоде менопаузы. Известно, что у женщин среднего возраста с нормальной массой тела содержание в плазме, как общего холестерина, так и липопротеидов низкой плотности ниже, чем у мужчин, а липопротеидов высокой плотности - выше. При наступлении менопаузы у женщин, по мере снижения продукции эстрогенов, увеличивается количество висцерального жира, уменьшается активность печеночной липазы, что сопровождается снижением уровня липопротеидов высокой плотности в крови [17].

Заключение. Таким образом, в нашем исследовании установлено, что абдоминальное ожирение у пожилых пациентов с СНпФВ ишемического генеза на фоне метаболического синдрома имеет прямую корреляцию с гиперлептинемией. Наличие признаков метаболического синдрома у пожилых больных СНпФВ ишемического генеза ассоциируется как с гиперинсулинемией, так и с инсулинорезистентностью, а липидный профиль крови отличается не только гиперхолестеринемией, но и гипертриглицеридемией, увеличением концентрации липопротеидов низкой плотности и снижением уровня липопротеидов высокой плотности. Установлены отдельные гендерные особенности у пожилых больных СНпФВ ишемического генеза и нарушением метаболизма. Так, у пожилых женщин с СНпФВ ишемического генеза на фоне МС отмечается наиболее выраженное по сравнению с мужчинами абдоминальное ожирение, ассоциированное с более высокими уровнями лептина крови и инсулинорезистентности, более высоким уровнем ЛПНП и более низким содержанием ЛПВП.

\section{Информация о финансировании}

Финансирование данной работы не проводилось.

\section{Financial support}

No financial support has been provided for this work.

\section{Конфликт интересов}

Автор заявляет об отсутствии конфликта интересов.

\section{Conflict of interests}

The author has no conflict of interest to declare.

\section{Список литературы}

1. Мареев ВЮ, Фомин ИВ, Агеев ФТ, и др. Клинические рекомендации. Хроническая сердечная недостаточность (ХCH). Сердечная Недостаточность. 2017;18(1);3-40. DOI: 10.18087/rhfj.2017.1.2346

2. Simmonds SJ, Cuijpers I, Heymans S, et al. Cellular and Molecular Differences between HFpEF and HFrEF: A Step Ahead in an Improved Pathological Understanding. Cells. 2020;9(1):242. DOI: https://doi.org/10.3390/cells9010242

3. Varounis C, Rallidis LS, Franco OH, et al. Prevalence of metabolic syndrome and association with burden of atherosclerotic disease in patients with stable coronary artery disease. Journal Current Medical Research and Opinion. 2016;32(6):1175-1181. DOI: https://doi.org/10.1185/03007995.2016.1163257

4. Драпкина ОМ, Шепель РН, Шальнова $\mathrm{CA}$, и др. Основные антропометрические индексы и сахарный диабет 2 типа в российской популяции. Рациональная фармакотерапия в кардиологии. 2018;14(5):725-732. DOI: 10.20996/1819-6446-2018-14-5-725-732

5. Полякова ЕА, Драганова АС, Колодина ДА, и др. Экспрессия гена лептина в эпикардиальной жировой ткани у мужчин с ишемической болезнью сердца. Артериальная ги- 
пертензия. 2017;23(6):488-497. DOI: https://doi.org/10.18705/1607-419X-2017-23-6488-497

6. Чумакова ГА, Отт АВ, Веселовская НГ, и др. Патогенетические механизмы лептинорезистентности. Российский кардиологический журнал. 2015;(4):107-110. DOI: https://doi.org/10.15829/1560-4071-2015-4-107110

7. Kyung WL, Dayeon S. Prospective Associations of Serum Adiponectin, Leptin, and Leptin-Adiponectin Ratio with Incidence of Metabolic Syndrome: The Korean Genome and Epidemiology Study. International Journal of Environmental Research and Public Health. 2020;17(9):3287.

DOI:

http://dx.doi.org/10.3390/ijerph17093287

8. Вавилова ТП, Плетень АП, Михеев РК. Биологическая роль адипокинов как маркеров патологических состояний. Вопросы питания. 2017;86(2):5-13. DOI: 10.24411/00428833-2017-00028

9. Цанава ИА, Шаронова ЛА, Вербовой А.Ф. Метаболический синдром и сердечнососудистые заболевания. РМЖ. Медицинское обозрение. 2017;11:785-789.

10.Meyer-Gerspach AC, Cajacob L, Riva D, et al. Mechanisms Regulating Insulin Response to Intragastric Glucose in Lean and Non-Diabetic Obese Subjects: A Randomized, Double-Blind, Parallel-Group Trial. PLoS One. 2016;11(3):e0150803.

DOI:

10.1371/journal.pone.0150803

11.Sung K, Park H, Kim M, et al. Metabolic markers associated with insulin resistance predict type 2 diabetes in Koreans with normal blood pressure or prehypertension. Cardiovascular Diabetology. 2016;15:47.

DOI: https://doi.org/10.1186/s12933-016-0368-7

12.Gui MH, Ling Y, Liu L, et al. Effect of Metabolic Syndrome Score, Metabolic Syndrome, and Its Individual Components on the Prevalence and Severity of Angiographic Coronary Artery Disease. Chin Med J (Engl). 2017;130(6):669677. DOI: 10.4103/0366-6999.201611

13.Братусь ВВ, Шумаков ВА, Талаева TB, и др. Нормализующее влияние триметазидина на компоненты синдрома инсулинорезистентности и функциональное состояние миокарда у пациентов с ишемической болезнью сердца. Украінский кардіологичний журнал. 2004;2:41-49.
14.Saad MF, Damani S, Gingerich RL, et al. Sexual dimorphism in plasma leptin concentration. The Journal of Clinical Endocrinology \& Metabolism. 1997;82(2):579-584. DOI: https://doi.org/10.1210/jcem.82.2.3739

15.Ткаченко ЛВ, Свиридова НИ, Складановская ТВ. Современное состояние проблемы избыточной массы тела и ожирения у женщин. Вестник Волгоградского государственного медицинского университета. 2017;4(64):3-7. DOI: 10.19163/1994-9480-20174(64)-3-7

16.Баянова Е.Г. Взаимосвязь между компонентами метаболического синдрома у больных ожирением. Международный эндокринологический журнал. 2017;2(13):209-12. DOI: 10.22141/2224-0721.13.2.2017.100615

17.Мычка ВБ, Толстов СН, Салов ИА. Особенности формирования метаболического синдрома у женщин в разные фазы климактерического периода. Российский кардиологический журнал. 2015;4(120):73-78. DOI: http://dx.doi.org/10.15829/1560-4071-2015-0473-78

\section{References}

1. Mareev VYu, Fomin IV, Ageev FT, et al. Clinical guidelines. Chronic heart failure (CHF). Russian Heart Failure Journal. 2017;18(1);3-40. Russian. DOI: 10.18087/rhfj.2017.1.2346

2. Simmonds SJ, Cuijpers I, Heymans S, et al. Cellular and Molecular Differences between HFpEF and HFrEF: A Step Ahead in an Improved Pathological Understanding. Cells. 2020;9(1):242. DOI: https://doi.org/10.3390/cells9010242

3. Varounis $\mathrm{C}$, Rallidis LS, Franco $\mathrm{OH}$, et al. Prevalence of metabolic syndrome and association with burden of atherosclerotic disease in patients with stable coronary artery disease. Journal Current Medical Research and Opinion. 2016;32(6):1175-1181. DOI: https://doi.org/10.1185/03007995.2016.1163257

4. Drapkina OM, Shepel RN, Shalnova $\mathrm{SA}$, et al. Basic Anthropometric Indices and Type 2 Diabetes Mellitus in Russian Population. Rational Pharmacotherapy in Cardiology. 2018;14(5):725-732. Russian. DOI: 10.20996/1819-6446-2018-14-5-725-732

5. Polyakova EA, Draganova AS, Kolodina DA, et al. Leptin gene expression in epicardial adipose tissue in males with coronary heart disease. Arterial Hypertension. 2017;23(6):488- 
497. Russian. DOI: https://doi.org/10.18705/1607419X-2017-23-6-488-497

6. Chumakova GA, Ott AV, Veselovskaya NG, et al. Pathogenetic mechanisms of leptin resistance. Russian Journal of Cardiology. 2015;(4):107-110. Russian. DOI: https://doi.org/10.15829/1560-4071-2015-4-107110

7. Kyung WL, Dayeon S. Prospective Associations of Serum Adiponectin, Leptin, and Leptin-Adiponectin Ratio with Incidence of Metabolic Syndrome: The Korean Genome and Epidemiology Study. International Journal of Environmental Research and Public Health. 2020;17(9):3287.

DOI: http://dx.doi.org/10.3390/ijerph17093287

8. Vavilova TP, Pleten' AP, Mikheev RK. Biological role of adipokines and their association with morbid conditions. Voprosy pitaniia [Problems of Nutrition]. 2017;86(2):5-13. Russian. DOI: 10.24411/0042-8833-2017-00028

9. Tsanava IA, Sharonova LA, Verbovoy AF. Metabolic syndrome and cardiovascular diseases. RMJ. 2017;11:785-789. Russian.

10. Meyer-Gerspach AC, Cajacob L, Riva $\mathrm{D}$, et al. Mechanisms Regulating Insulin Response to Intragastric Glucose in Lean and Non-Diabetic Obese Subjects: A Randomized, Double-Blind, Parallel-Group Trial. PLoS One. 2016;11(3):e0150803. DOI: 10.1371/journal.pone.0150803

11.Sung K, Park H, Kim M, et al. Metabolic markers associated with insulin resistance predict type 2 diabetes in Koreans with normal blood pressure or prehypertension. Cardiovascular Diabetology. 2016;15:47.

DOI: https://doi.org/10.1186/s12933-016-0368-7

12.Gui MH, Ling Y, Liu L, et al. Effect of Metabolic Syndrome Score, Metabolic Syndrome, and Its Individual Components on the Prevalence and Severity of Angiographic Coronary Artery Disease. Chin Med J (Engl). 2017;130(6):669677. DOI: 10.4103/0366-6999.201611

13. Bratus VV, Shumakov VA, Talaeva TV, et al. Influence of trimetazidine on insulin resistance syndrome components and cardiac functional abilities in patients with ischemic heart disease. Ukrainian journal of cardiology. 2004;2:4149. Russian.
14.Saad MF, Damani S, Gingerich RL, et al. Sexual dimorphism in plasma leptin concentration. The Journal of Clinical Endocrinology \& Metabolism. 1997;82(2):579-584. DOI: https://doi.org/10.1210/jcem.82.2.3739

15.Tkachenko LV, Sviridova NI, Skladanovskaya TV. Current state of the problem of overweight and obesity in women. Vestnik Volgogradskogo gosudarstvennogo medicinskogo universiteta. 2017;4(64):3-7. Russian. DOI: 10.19163/1994-9480-2017-4(64)-3-7

16.Bayanova EG. Interconnection between metabolic syndrome components in obese patients. Mezhdunarodnyi Endokrinologicheskii Zhurnal. 2017;13:209-12. Russian. DOI: 10.22141/2224-0721.13.2.2017.100615

17. Mychka VB, Tolstov SN, Salov IA. Specifics of metabolic syndrome pathophysiology in women at different phases of climacteric period. Russian Journal of Cardiology. 2015;4(120):73-78. Russian. DOI: https://doi.org/10.15829/1560-4071-2015-4-73-78

Статья поступила в редакцию 08 марта 2020 г. Поступила после доработки 03 мая 2020 г. Принята к печати 26 мая 2020 г.

Received 8 March 2020

Revised 3 May 2020

Accepted 26 May 2020

\section{Информация об авторе}

Елена Владимировна Гостева, кандидат медицинских наук, доцент, доцент кафедры пропедевтики внутренних болезней ФГБОУ ВО «Воронежский государственный медицинский университет им. Н.Н. Бурденко» Минздрава России, E-mail: pvb.gosteva@mail.ru, ORCID: 0000-0002-8771-2558.

\section{Information about the author}

Elena V. Gosteva, Candidate of Medical Sciences, Associate Professor, Associate Professor of the Department of Propaedeutics of Internal Diseases of the Burdenko Voronezh State Medical University, E-mail: pvb.gosteva@mail.ru, ORCID: 00000002-8771-2558. 